\title{
Development of Multi-Chroic MKIDs for Next-Generation CMB Polarization Studies
}

\author{
B. R. Johnson ${ }^{\mathrm{a}}$. D. Flanigan ${ }^{\mathrm{a}}$ - M. H. Abitbol $^{\mathrm{a}}$. \\ P. A. R. Ade ${ }^{\mathrm{b}}$. S. Bryan ${ }^{\mathrm{c}} \cdot$ H.-M. Cho ${ }^{\mathrm{g}}$. \\ R. Datta ${ }^{\mathrm{e}, \mathrm{h}} \cdot$ P. Day ${ }^{\mathrm{f}} \cdot$ S. Doyle $^{\mathrm{b}} \cdot$ K. Irwin ${ }^{\mathrm{d}, \mathrm{g}}$. \\ G. Jones ${ }^{\mathrm{a}} \cdot$ D. $\mathbf{L i}^{\mathrm{g}} \cdot$ P. Mauskopf ${ }^{\mathrm{c}}$. \\ H. McCarrick ${ }^{\mathrm{a}}$ - J. McMahon ${ }^{\mathrm{e}}$. A. Miller ${ }^{\mathrm{a}}$.

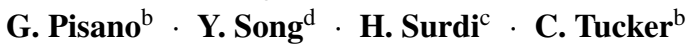 \\ the date of receipt and acceptance should be inserted later
}

\begin{abstract}
We report on the status of an ongoing effort to develop arrays of horn-coupled, polarization-sensitive microwave kinetic inductance detectors (MKIDs) that are each sensitive to two spectral bands between 125 and $280 \mathrm{GHz}$. These multi-chroic MKID arrays are tailored for next-generation, large-detector-count experiments that are being designed to simultaneously characterize the polarization properties of both the cosmic microwave background (CMB) and Galactic dust emission. We present our device design and describe laboratory-based measurement results from two 23-element prototype arrays. From dark measurements of our first engineering array we demonstrated a multiplexing factor of 92 , showed the resonators respond to bath temperature changes as expected, and found that the fabrication yield was $100 \%$. From our first optically-loaded array we found the MKIDs respond to millimeter-wave pulses; additional optical characterization measurements are ongoing. We end by discussing our plans for scaling up this technology to kilo-pixel arrays over the next two years.
\end{abstract}

Keywords MKIDs, multi-chroic, CMB, polarization

\section{Introduction}

In this paper we report on the status of an ongoing effort to develop arrays of polarizationsensitive, microwave kinetic inductance detectors (MKIDs) for future cosmic microwave background $(\mathrm{CMB})$ studies $\frac{12}{12}$. The array elements we are developing are each sensitive to both polarizations in two spectral bands, so there are four MKIDs per array element. These multi-chroic MKIDs are designed to help separate foreground signals from CMB signals. One of the spectral bands is for detecting the CMB, so it is centered on $150 \mathrm{GHz}$, which is near the peak of the CMB blackbody spectrum. The second spectral band is primarily for

a) Department of Physics, Columbia University, New York, NY, 10027, USA;

b) School of Physics \& Astronomy, Cardiff University, Cardiff, CF243AA, UK;

c) School of Earth and Space Exploration, Arizona State University, Tempe, AZ, 85287, USA;

d) Department of Physics, Stanford University, Stanford, CA, 94305-4085, USA;

e) Department of Physics, University of Michigan, Ann Arbor, MI, 48103, USA;

f) NASA, Jet Propulsion Lab, Pasadena, CA, 91109, USA;

g) SLAC National Accelerator Laboratory, Menlo Park, CA 94025, USA

h) NASA Goddard Space Flight Center, Mail Code 665, Greenbelt, MD 20771, USA

E-mail: bradley.johnson@columbia.edu 

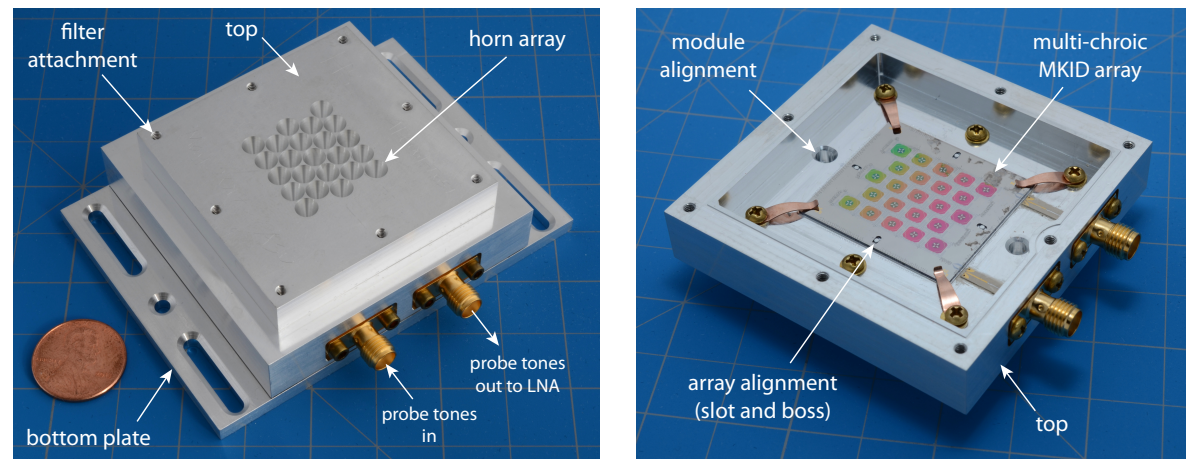

Fig. 1 Left: Photograph of the 23-element multi-chroic MKID module that is described in Section 2 The module consists of an aluminum enclosure and an MKID array, which is mounted inside. Right: The top of the enclosure contains 23 conical horns and the detector array, which has a total of 92 MKIDs. In this photo, the entrance apertures of the horns are facing down and the bottom plate is removed. The alignment of the MKID array is controlled using four bosses that are machined in the aluminum enclosure and four slots that are etched in the silicon with a deep-reactive ion etch (DRIE) process. This configuration keeps the array aligned while allowing for differential thermal contraction. The slots are nominally $10 \mu \mathrm{m}$ wider than the bosses, and this gap sets the lateral alignment accuracy for the MKID array. All of the MKIDs are read out with a single transmission line, so the multiplexing factor for this detector system is 92 .

detecting Galactic dust signals, so it is centered on $235 \mathrm{GHz}$, where Galactic dust emission is brighter than the CMB.

MKIDs are planar superconducting GHz resonators. The kinetic inductance and dissipation of the superconducting film depend on the quasiparticle density. When sufficiently energetic photons $\Phi^{\dagger}$ are absorbed by the MKID, Cooper pairs break, causing an increase in the quasiparticle density, a decrease in the resonant frequency, and a decrease in the resonator quality factor ${ }^{3}$. These changes can be detected by monitoring the amplitude and phase of a probe tone that drives the resonator at its nominal resonant frequency. Each MKID resonator is given a unique resonant frequency, so hundreds to thousands of detectors in an array can be read out on a single transmission line. The detector architecture we describe in this paper is designed to be well suited for next-generation CMB studies that require on the order of five hundred thousand detectors 4 .

Our development program is currently focused on building 23-element prototype multichroic MKID modules and testing them in the laboratory. We present our module design in Section 2. To date, we have fabricated and tested one module and one engineering array. Our measurement results are presented in Section 3 Finally, over the next two years we will scale up our module design and build a kilo-pixel array. These future plans are discussed in Section 4

\section{Methods}

The 23-element prototype modules we are currently building are composed of an MKID array fabricated on a silicon-on-insulator (SOI) wafer that is mounted inside a two-piece aluminum enclosure. The top of the aluminum enclosure is a monolithic horn plate that also serves as the mounting surface for the MKID array. The bottom plate, which closes the module, contains backshorts, which are used to optimize the millimeter-wave coupling. The

\footnotetext{
${ }^{\dagger}$ If $v>2 \Delta / h \cong 74 \mathrm{GHz} \times\left(T_{c} / 1 \mathrm{~K}\right)$, the photons will break Cooper pairs. Here, $\Delta$ is the superconducting
} gap, $h$ is Planck's constant, and $T_{C}$ is the superconducting transition temperature of the film. 


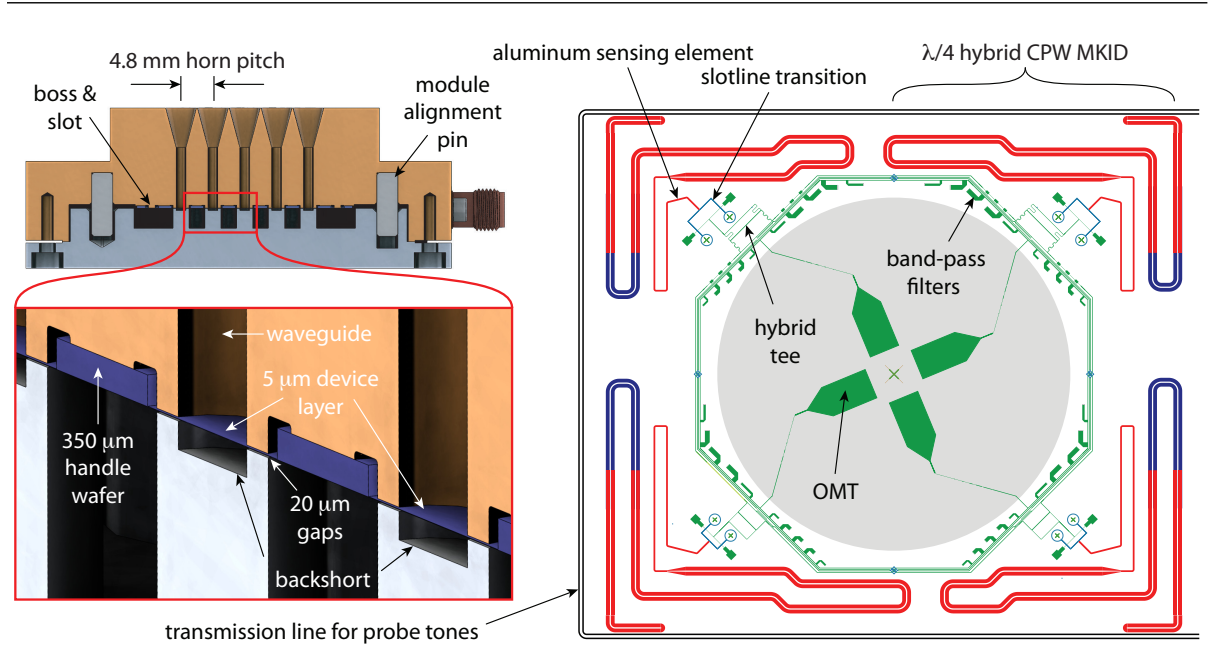

Fig. 2 Left: A cross-sectional view of the multi-chroic MKID module. Each conical horn feeds a cylindrical waveguide, which in turn, feeds an array element. The array is fabricated on a silicon-on-insulator (SOI) wafer. Backshorts are used to optimize the millimeter-wave coupling. Right: A scale drawing of one array element. The cylindrical waveguide feeds the probe pairs in the orthomode transducer (OMT). Sky signals are filtered with a microstrip band-pass and then coupled to the coplanar waveguide (CPW) MKID with a novel slotline transition 6 . Each array element has four MKIDs - two polarizations in each of the two spectral bands.

aluminum enclosure is machined with a high-precision Kern 44 computer numeric control (CNC) mill, so the top and the bottom of the enclosure are aligned to within a few microns. A photograph of the module is shown in Figure 1, and the array-element design is shown in Figure 2

A conical horn with a $4.66 \mathrm{~mm}$ diameter aperture and a 15 deg flare angle is used to feed each array element in the module. We chose to use conical horns in the prototype module for convenience, and we will switch to profiled horns in the future ${ }^{7}$. Light emerging from the cylindrical waveguide at the back of the conical horn is coupled to a broadband planar orthomode transducer (OMT). The waveguide diameter is $1.49 \mathrm{~mm}$ and we made it approximately $9 \mathrm{~mm}$ long to ensure evanescent low-frequency modes do not reach the detectors. The OMT separates the two polarizations of the incoming millimeter-wave signal using two waveguide probe pairs ${ }^{8}$. A choke around the exit aperture of the cylindrical waveguide improves the waveguide/OMT coupling efficiency by minimizing the fields that leak laterally into the module along the array.

The two spectral bands (125 to $170 \mathrm{GHz}$ and 190 to $280 \mathrm{GHz}$ ) are defined by fivepole resonant-stub microstrip band-pass filters (see Figure 3). The output of each waveguide probe is coplanar waveguide (CPW). Therefore, a broadband CPW-to-microstrip transition is first used to connect the waveguide probes to the filter circuit. This transition is composed of seven alternating sections of CPW and microstrip. The signals from each probe pair within a single spectral band are then combined using a $180^{\circ}$ hybrid. The dimensions of all of these millimeter-wave circuit components are given in the literature ${ }^{9}$. The $\mathrm{TE}_{11}$ waveguide mode ultimately couples to the difference port on the $180^{\circ}$ hybrid, while higherorder modes couple to the sum port $2[8$. Therefore, to ensure single-moded performance over the required bandwidth ratio of $2.25: 1$, signals at the sum port of the $180^{\circ}$ hybrid are routed to a termination resistor and discarded, while signals at the difference port are sent to the MKID using a broadband coupling circuit ${ }^{\sqrt[6]{6}}$ (described below). 


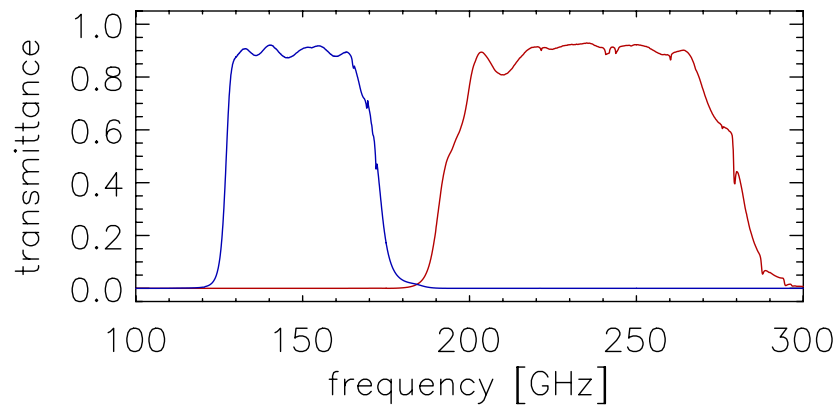

Fig. 3 Simulated spectral bands in each of the multi-chroic MKID array elements 296 . The electromagnetic simulations that were used to compute these curves start at the CPW output of the OMT, include all of the millimeter-wave circuit components, and end in the absorbing element of the MKID (see Section 2). The expected absorption efficiency is approximately $90 \%$ across both the $150 \mathrm{GHz}$ and the $235 \mathrm{GHz}$ spectral bands. A metal-mesh low-pass filter with an edge near $360 \mathrm{GHz}$ will be used to reject high-frequency, out-ofband radiation. The metal-mesh filter will be mounted outside the module in front of the horn apertures (see Figure 1. The transmittance of this filter and any frequency-dependent effects from the waveguide-to-OMT coupling are not included in these simulations.

The MKIDs are $\lambda / 4$ resonators composed of two sections of CPW in series. The center line of the resonator is electrically grounded at one end and capacitively coupled at the other end to the transmission line used to read out the array. The section of CPW at the grounded end of the resonator is the absorptive section, and the center line here is made from aluminum. Our thin-film aluminum has $T_{c}=1.4 \mathrm{~K}$, so photons with frequencies greater than approximately $100 \mathrm{GHz}$ will be energetic enough to break Cooper pairs in the aluminum. It is desirable to make the volume of this absorber as small as possible to increase the responsivity. As a starting point, we chose the dimensions of our absorbing element to be $4 \mu \mathrm{m}$ wide, $40 \mathrm{~nm}$ thick, $2.1 \mathrm{~mm}$ long for the $150 \mathrm{GHz}$ spectral band and $2.7 \mathrm{~mm}$ long for the $235 \mathrm{GHz}$ spectral band. The absorbing element is longer for the $235 \mathrm{GHz}$ spectral band because we anticipate there will be more loading from the sky, so the volume needs to be larger. Simulations show that with these lengths, approximately $90 \%$ of the millimeterwave power from the sky will be absorbed in the aluminum and break Cooper pairs ${ }^{2}$. The gaps between the aluminum center line and the ground plane are $5 \mu \mathrm{m}$ wide. The second section of center line is made from a niobium-over-aluminum bilayer, which has $T_{c}=8.3 \mathrm{~K}$. The photons in our two spectral bands will not be energetic enough to break Cooper pairs in this section of the resonator. This second section of CPW is used to tune the resonant frequency of the MKID, so the length varies from detector to detector; the length range is 8.8 to $10.4 \mathrm{~mm}$. The end of the resonator near the readout transmission line has the largest electric fields and is therefore most susceptible to two-level system (TLS) effects $\frac{10[11}{}$. To minimize any TLS effects, the gap to the ground plane in the CPW should be wide. As a starting point, we made the niobium center line $10 \mu \mathrm{m}$ wide and the gap to the ground plane $30 \mu \mathrm{m}$ wide. This $\sim 10 \mathrm{~mm}$ total length of CPW produces resonant frequencies near $3 \mathrm{GHz}$. Hybrid CPW MKIDs like these have been shown to be photon-noise limited over a wide range of millimeter and sub-millimeter wavelengths with optical loading levels well under $1 \mathrm{pW} 12[13$.

To couple the microstrip output of the $180^{\circ}$ hybrid to the MKID, the power is first evenly divided in-phase onto two microstrips, each with twice the impedance of the microstrip from the $180^{\circ}$ hybrid. Each branch feeds a standard broadband microstrip-to-slotline transition, where the slotline is formed in the niobium ground plane that is common to the microstrip 

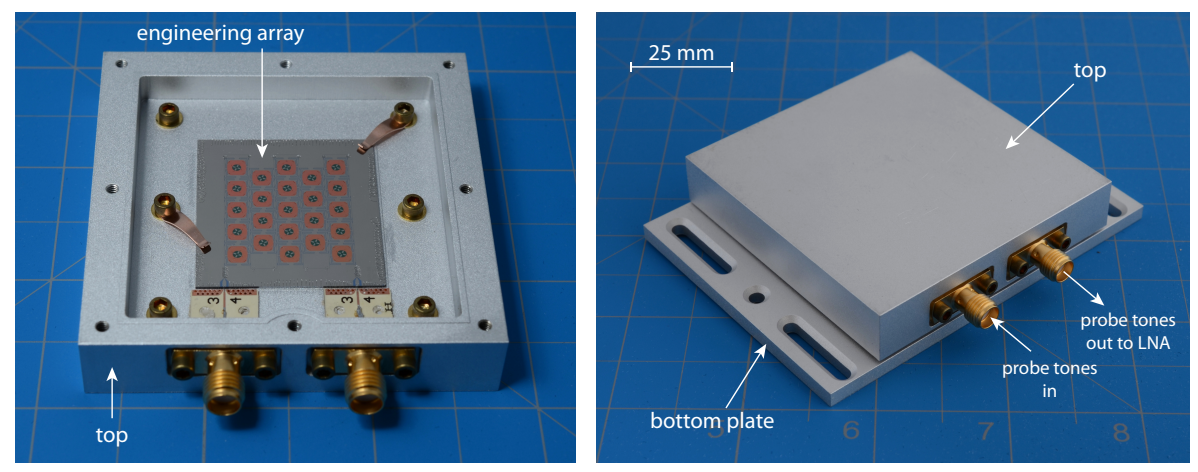

Fig. 4 Left: Photograph of the engineering array mounted inside a separate aluminum enclosure. Right: This second enclosure does not have any conical horns, so after the bottom plate is installed and the module is sealed, the MKIDs are not externally illuminated. Characterization measurements of the resonators in this engineering array are presented in Section 3

circuit and the MKID. The two slotlines are then brought together and become the CPW gaps in the aluminum section of the MKID.

The MKID arrays are fabricated on SOI wafers $100 \mathrm{~mm}$ in diameter. Each SOI wafer consists of a $5 \mu \mathrm{m}$ thick float-zone silicon ( $>10 \mathrm{k} \Omega \mathrm{cm}$ resistivity) device layer and a $350 \mu \mathrm{m}$ thick silicon handle wafer held together by a $0.5 \mu \mathrm{m}$ thick buried oxide layer. An aluminum/niobium bilayer is first deposited on the device layer. The aluminum is $40 \mathrm{~nm}$ thick and the niobium is $200 \mathrm{~nm}$ thick. This bilayer is patterned to produce the OMTs, the MKIDs, and the ground plane. A $350 \mathrm{~nm}$ thick film of silicon nitride $\left(\mathrm{SiN}_{x}\right)$ is deposited on top of the bilayer, followed by a $400 \mathrm{~nm}$ thick niobium film. The $\mathrm{SiN}_{x}$ serves as the electrically insulating dielectric material in the microstrip, and the niobium film is patterned to form the microstrip circuit that includes the band-pass filters and the $180^{\circ}$ hybrids. Our design uses cross-unders ${ }^{14}$ in the microstrip circuit rather than cross-overs, which decreases the number of required fabrication steps. A gold film is deposited and patterned on top of the $\mathrm{SiN}_{x}$ to construct the aforementioned termination resistor at the sum port of the $180^{\circ}$ hybrid. The $\mathrm{SiN}_{x}$ is removed near the MKIDs to reduce loss and two-level system (TLS) noise ${ }^{15}$. The bilayer niobium is removed from the $\sim 2 \mathrm{~mm}$ long sensing section of the center line of the MKID leaving only the aluminum. To improve the optical coupling and to minimize TLS noise, the thick silicon handle wafer and the buried oxide layer underneath the OMT and the high-field section of the MKID are removed using deep reactive ion etching (DRIE).

\section{Results}

We first produced an engineering array on a monolithic $500 \mu \mathrm{m}$ thick float-zone silicon wafer to test a majority of the processing steps (see Figure 4). This engineering array is not optimized for millimeter-wave coupling because the substrate is too thick. Therefore, we mounted it in a second aluminum enclosure with no horns, chokes, or backshorts and ran some resonator tests where the MKIDs were not illuminated.

In these tests, we drove the resonators with probe tones via the readout transmission line, and we measured the amplitude and phase of the emerging waveforms. These tests were done over a range of module temperatures between $85 \mathrm{mK}$ and $400 \mathrm{mK}$. The details of the readout system we used are given in the literature ${ }^{2}$. By sweeping the frequency of the probe tone from 1.8 to $4.0 \mathrm{GHz}$, we measured the complex forward transmission $\left(S_{21}\right)$. The 

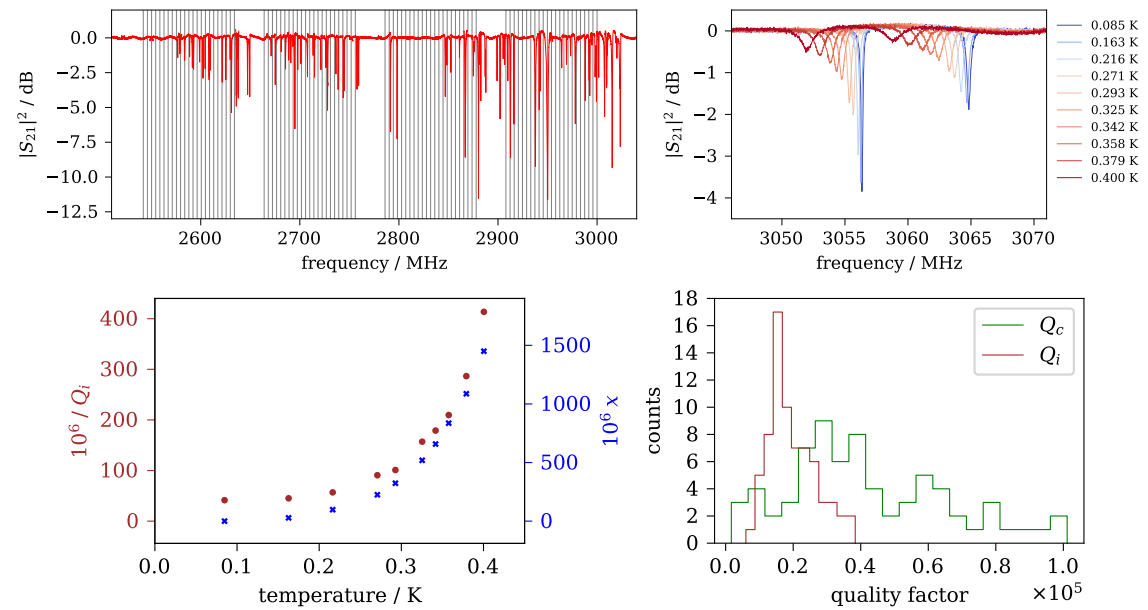

Fig. 5 Measurements of the resonators in the engineering array (see Figure 4). Top Left: Measured complex forward transmission $\left(S_{21}\right)$ as a function of frequency. The vertical gray lines indicate the designed resonant frequencies. For this measurement, the module temperature was $200 \mathrm{mK}$. Top Right: Measured $S_{21}$ for two resonators as a function of temperature. The resonant frequency and quality factor decrease when the temperature increases, as expected. Bottom Left: Measured fractional frequency shift $x$ and inverse internal quality factor $Q_{i}^{-1}$ as a function of module temperature for a $2695 \mathrm{MHz}$ resonator. The internal quality factor of the resonator is related to $Q$ and $Q_{c}$ as $Q^{-1}=Q_{i}^{-1}+Q_{c}^{-1}$. Bottom Right: Histogram of $Q_{i}$ and $Q_{c}$ measured at $85 \mathrm{mK}$. Data from 71 of the 92 resonators are included in the histogram. Data from the additional 21 resonators require more careful fitting, in part because many of them partially overlap in frequency, so we did not include them in this initial study. Future arrays will include lithographed connections between the ground planes on opposite sides of the transmission line CPW, which should reduce the scatter in resonance frequencies.

$S_{21}$ data is shown in the top panels of Figure 5 . To characterize the resonances, data from each resonator was fit to the equation 16

$$
S_{21}=1-\frac{Q}{Q_{c}}\left(\frac{1}{1+2 j x Q}\right),
$$

where $Q_{c}$ is the complex coupling quality factor, $Q$ is the resonator quality factor, $x=$ $1-f / f_{0}$ is the fractional frequency shift, $f$ is the variable resonant frequency, $f_{0}$ is the reference resonant frequency, and we have omitted an overall phase term for clarity. This measurement revealed (i) the resonant frequencies of all 92 resonators in the array, so the MKID fabrication yield was $100 \%$, and (ii) the resonant frequency and quality factor of the resonator changed with bath temperature as expected.

Characterization measurements of our first optically-alive module (see Figure 11) are underway 1 . These measurement results will be published in the future. However, in Figure 6 we show some preliminary data: the MKIDs are responding to millimeter-waves generated with an electronic source that is rapidly switched on and off with a PIN diode. For this measurement, we used a cryogenic test system that was previously used for lumped-element kinetic inductance detector studies, and the details of this test system are described in the literature $\frac{17 \mid 18}{}$.

\section{Discussion}

Over the next two years, we will develop a hexagonal multi-chroic MKID module that contains 169 array elements (676 MKIDs). Multiple modules can therefore be arranged to form 

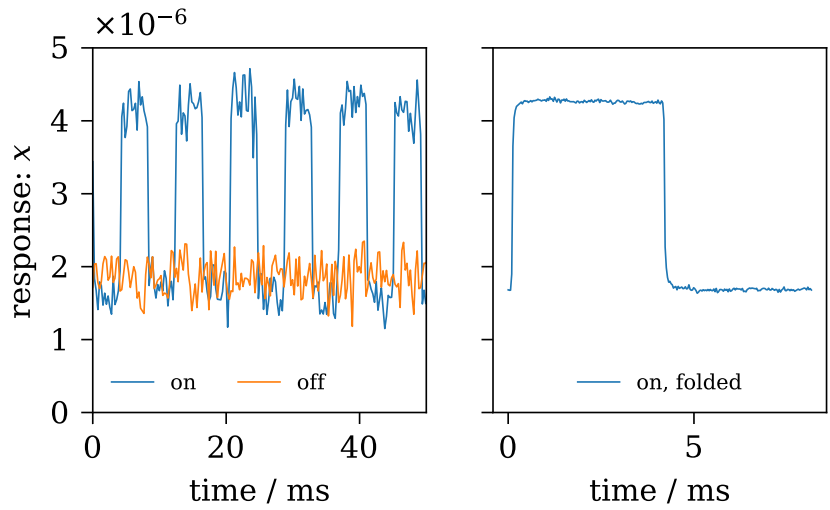

Fig. 6 Millimeter-waves detected with our first multi-chroic MKID module (see Figure 1). The incoherent broadband (140 to $160 \mathrm{GHz}$ ) millimeter-wave pulses were generated using an electronic source 1718 . Left: Time-ordered data with the millimeter-wave pulses both on and off. Right: To clearly show the shape of the detected pulse, many pulses have been folded down to one period by averaging samples separated in time by an integer number of pulse periods.
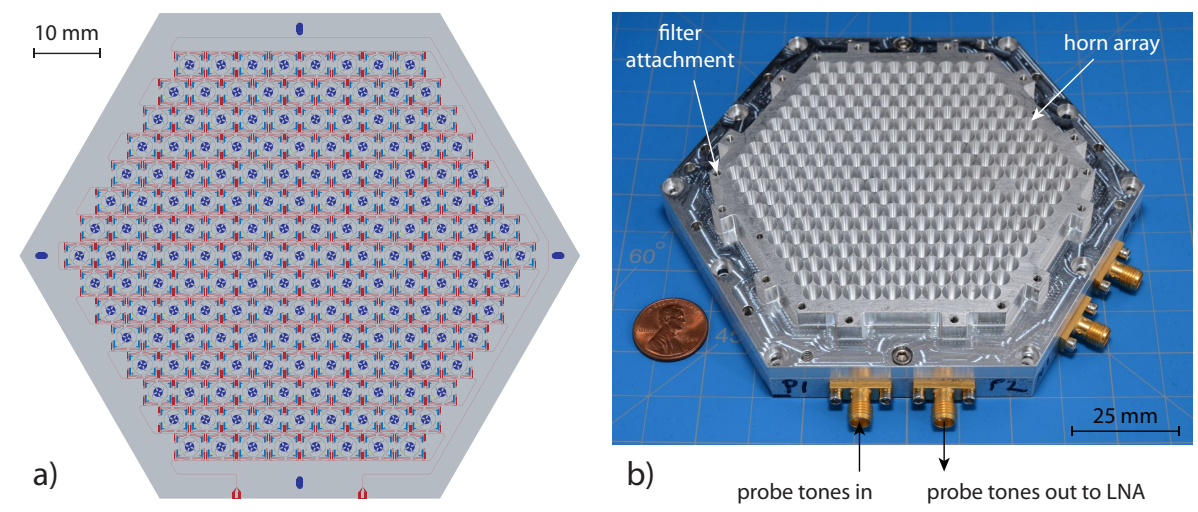

Fig. 7 Left: Scale drawing of the 169-element multi-chroic MKID array we are developing. Right: Photograph of a hexagonal aluminum module. This particular module has 271 horns and it was made for lumpedelement kinetic inductance detectors $\frac{17}{}$, but the multi-chroic MKID module we will build will look similar.

a multi-kilo-pixel array. The array design and a concept enclosure are shown in Figure 7. For this new module we will switch to profiled horns and replace the aluminum films in the array with aluminum-manganese (AlMn) films. AlMn has a tunable $T_{c}$ that can be decreased below 1.4 K. Reducing the sensor $T_{c}$ increases the average number of Cooper pairs broken by each photon in our two spectral bands, and thus decreases the relative contribution of recombination noise ${ }^{18}$. Members of our collaboration pioneered the use of AlMn as a material for transition edge sensor (TES) bolometers $\frac{19}{}$, and we have already shown that AlMn works as a high-Q resonator material 20 .

Acknowledgements This work is supported by NSF grants AST-1509211 and AST-1711160 for Johnson; AST-1509078 and AST-1711242 for Mauskopf; AST-1506074 and AST-1710624 for Irwin. McCarrick is supported by a NASA Earth and Space Sciences Fellowship. We thank the Xilinx University Program for donating the FPGA hardware and software tools that were used in the readout system. 


\section{References}

1. D. Flanigan. Kinetic Inductance Detectors for Measuring the Polarization of the Cosmic Microwave Background. PhD thesis, Columbia University, 2018.

2. Bradley R. Johnson, Daniel Flanigan, Maximilian H. Abitbol, Peter A. R. Ade, Sean Bryan, Hsiao-Mei Cho, Rahul Datta, Peter Day, Simon Doyle, Kent Irwin, Glenn Jones, Sarah Kernasovskiy, Dale Li, Philip Mauskopf, Heather McCarrick, Jeff McMahon, Amber Miller, Giampaolo Pisano, Yanru Song, Harshad Surdi, and Carole Tucker. Polarization-sensitive multi-chroic MKIDs. Proc. SPIE, 9914:99140X, 2016.

3. P. K. Day, H. G. LeDuc, B. A. Mazin, A. Vayonakis, and J. Zmuidzinas. A broadband superconducting detector suitable for use in large arrays. Nature, 425:817-821, 2003.

4. M. H. Abitbol, Z. Ahmed, D. Barron, R. Basu Thakur, A. N. Bender, B. A. Benson, C. A. Bischoff, S. A. Bryan, J. E. Carlstrom, C. L. Chang, D. T. Chuss, K. T. Crowley, A. Cukierman, T. de Haan, M. Dobbs, T. Essinger-Hileman, J. P. Filippini, K. Ganga, J. E. Gudmundsson, N. W. Halverson, S. Hanany, S. W. Henderson, C. A. Hill, S.-P. P. Ho, J. Hubmayr, K. Irwin, O. Jeong, B. R. Johnson, S. A. Kernasovskiy, J. M. Kovac, A. Kusaka, A. T. Lee, S. Maria, P. Mauskopf, J. J. McMahon, L. Moncelsi, A. W. Nadolski, J. M. Nagy, M. D. Niemack, R. C. O’Brient, S. Padin, S. C. Parshley, C. Pryke, N. A. Roe, K. Rostem, J. Ruhl, S. M. Simon, S. T. Staggs, A. Suzuki, E. R. Switzer, O. Tajima, K. L. Thompson, P. Timbie, G. S. Tucker, J. D. Vieira, A. G. Vieregg, B. Westbrook, E. J. Wollack, K. W. Yoon, K. S. Young, and E. Y. Young. CMB-S4 Technology Book, First Edition. arXiv:1706.02464, 2017.

5. K. N. Abazajian, P. Adshead, Z. Ahmed, S. W. Allen, D. Alonso, K. S. Arnold, C. Baccigalupi, J. G. Bartlett, N. Battaglia, B. A. Benson, C. A. Bischoff, J. Borrill, V. Buza, E. Calabrese, R. Caldwell, J. E. Carlstrom, C. L. Chang, T. M. Crawford, F.-Y. Cyr-Racine, F. De Bernardis, T. de Haan, S. di Serego Alighieri, J. Dunkley, C. Dvorkin, J. Errard, G. Fabbian, S. Feeney, S. Ferraro, J. P. Filippini, R. Flauger, G. M. Fuller, V. Gluscevic, D. Green, D. Grin, E. Grohs, J. W. Henning, J. C. Hill, R. Hlozek, G. Holder, W. Holzapfel, W. Hu, K. M. Huffenberger, R. Keskitalo, L. Knox, A. Kosowsky, J. Kovac, E. D. Kovetz, C.-L. Kuo, A. Kusaka, M. Le Jeune, A. T. Lee, M. Lilley, M. Loverde, M. S. Madhavacheril, A. Mantz, D. J. E. Marsh, J. McMahon, P. D. Meerburg, J. Meyers, A. D. Miller, J. B. Munoz, H. N. Nguyen, M. D. Niemack, M. Peloso, J. Peloton, L. Pogosian, C. Pryke, M. Raveri, C. L. Reichardt, G. Rocha, A. Rotti, E. Schaan, M. M. Schmittfull, D. Scott, N. Sehgal, S. Shandera, B. D. Sherwin, T. L. Smith, L. Sorbo, G. D. Starkman, K. T. Story, A. van Engelen, J. D. Vieira, S. Watson, N. Whitehorn, and W. L. Kimmy Wu. CMB-S4 Science Book, First Edition. arXiv:1610.02743, 2016.

6. H. Surdi. Applications of Kinetic Inductance: Parametric Amplifier \& Phase Shifter, 2DEG Coupled Co-planar Structures \& Microstrip to Slotline Transition at RF Frequencies. Master's thesis, ARIZONA STATE UNIVERSITY, 2016.

7. P. Kittara, J. Leech, G. Yassin, B. K. Tan, A. Jiralucksanawong, and S. Wangsuya. High performance smooth-walled feed horns for focal plane arrays. In W. Wild, editor, Ninteenth International Symposium on Space Terahertz Technology, page 346, April 2008.

8. R. Datta, J. Austermann, J. A. Beall, D. Becker, K. P. Coughlin, S. M. Duff, P. A. Gallardo, E. Grace, M. Hasselfield, S. W. Henderson, G. C. Hilton, S. P. Ho, J. Hubmayr, B. J. Koopman, J. V. Lanen, D. Li, J. McMahon, C. D. Munson, F. Nati, M. D. Niemack, L. Page, C. G. Pappas, M. Salatino, B. L. Schmitt, A. Schillaci, S. M. Simon, S. T. Staggs, J. R. Stevens, E. M. Vavagiakis, J. T. Ward, and E. J. Wollack. Design and Deployment of a Multichroic Polarimeter Array on the Atacama Cosmology Telescope. 
J. Low Temp. Phys., 184(3):568-575, 2016.

9. R. Datta. The First Multichroic Receiver and Results from ACTPol. $\mathrm{PhD}$ thesis, University of Michigan, 2016.

10. J. Gao, M. Daal, A. Vayonakis, S. Kumar, J. Zmuidzinas, B. Sadoulet, B. A. Mazin, P. K. Day, and H. G. Leduc. Experimental evidence for a surface distribution of twolevel systems in superconducting lithographed microwave resonators. App. Phys. Lett., 92(15):152505, 2008.

11. J. Gao, M. Daal, J. M. Martinis, A. Vayonakis, J. Zmuidzinas, B. Sadoulet, B. A. Mazin, P. K. Day, and H. G. Leduc. A semiempirical model for two-level system noise in superconducting microresonators. Appl. Phys. Lett., 92(21):212504, 2008.

12. R. M. J. Janssen, J. J. A. Baselmans, A. Endo, L. Ferrari, S. J. C. Yates, A. M. Baryshev, and T. M. Klapwijk. Performance of hybrid NbTiN-Al microwave kinetic inductance detectors as direct detectors for sub-millimeter astronomy. Proc. SPIE, 9153:91530T, 2014.

13. S. J. C. Yates, J. J. A. Baselmans, A. Endo, R. M. J. Janssen, L. Ferrari, P. Diener, and A. Baryshev. Photon noise limited radiation detection with lens-antenna coupled microwave kinetic inductance detectors. Appl. Phys. Lett., 99(7):073505, 2011.

14. S. M. Duff, J. Austermann, J. A. Beall, D. Becker, R. Datta, P. A. Gallardo, S. W. Henderson, G. C. Hilton, S. P. Ho, J. Hubmayr, B. J. Koopman, D. Li, J. McMahon, F. Nati, M. D. Niemack, C. G. Pappas, M. Salatino, B. L. Schmitt, S. M. Simon, S. T. Staggs, J. R. Stevens, J. Van Lanen, E. M. Vavagiakis, J. T. Ward, and E. J. Wollack. Advanced ACTPol Multichroic Polarimeter Array Fabrication Process for $150 \mathrm{~mm}$ Wafers. J. Low Temp. Phys., 184(3):634-641, 2016.

15. Jonas Zmuidzinas. Superconducting Microresonators: Physics and Applications. Annu. Rev. Condens. Matter Phys., 3(1):169-214, 2012.

16. M. S. Khalil, M. J. A. Stoutimore, F. C. Wellstood, and K. D. Osborn. An analysis method for asymmetric resonator transmission applied to superconducting devices. $J$. Appl. Phys., 111(5):054510, 2012.

17. H. McCarrick, G. Jones, B. R. Johnson, M. H. Abitbol, P. A. R. Ade, S. Bryan, P. Day, T. Essinger-Hileman, D. Flanigan, H. G. Leduc, M. Limon, P. Mauskopf, A. Miller, and C. Tucker. Design and performance of dual-polarization lumped-element kinetic inductance detectors for millimeter-wave polarimetry. A\&A, 610:A45, February 2018.

18. D. Flanigan, H. McCarrick, G. Jones, B. R. Johnson, M. H. Abitbol, P. Ade, D. Araujo, K. Bradford, R. Cantor, G. Che, P. Day, S. Doyle, C. B. Kjellstrand, H. Leduc, M. Limon, V. Luu, P. Mauskopf, A. Miller, T. Mroczkowski, C. Tucker, and J. Zmuidzinas. Photon noise from chaotic and coherent millimeter-wave sources measured with horn-coupled, aluminum lumped-element kinetic inductance detectors. Appl. Phys. Lett., 108(8):083504, 2016.

19. Dale Li, Jason E. Austermann, James A. Beall, Daniel T. Becker, Shannon M. Duff, Patricio A. Gallardo, Shawn W. Henderson, Gene C. Hilton, Shuay-Pwu Ho, Johannes Hubmayr, Brian J. Koopman, Jeffrey J. McMahon, Federico Nati, Michael D. Niemack, Christine G. Pappas, Maria Salatino, Benjamin L. Schmitt, Sara M. Simon, Suzanne T. Staggs, Jeff Lanen, Jonathan T. Ward, and Edward J. Wollack. AlMn Transition Edge Sensors for Advanced ACTPol. J. Low Temp. Phys., 184(1):66-73, 2016.

20. G. Jones, B. R. Johnson, M. H. Abitbol, P. A. R. Ade, S. Bryan, H.-M. Cho, P. Day, D. Flanigan, K. D. Irwin, D. Li, P. Mauskopf, H. McCarrick, A. Miller, Y. R. Song, and C. Tucker. High quality factor manganese-doped aluminum lumped-element kinetic inductance detectors sensitive to frequencies below $100 \mathrm{GHz}$. Appl. Phys. Lett., 110(22):222601, 2017. 